Trauma Surgery \& Acute Care Open

\title{
New device for temporary hemorrhage control in penetrating injuries to the ventricles
}

\author{
Joao Baptista Rezende-Neto, ${ }^{1}$ Howard Leong-Poi, ${ }^{2}$ Sandro Rizoli, ${ }^{3}$ Andrew Beckett ${ }^{4}$
}

\begin{abstract}
${ }^{1}$ Department of Surgery, St. Michael's Hospital University of Toronto, Toronto, Ontario, Canada

${ }^{2}$ Department of Medicine, St. Michael's Hospital University of Toronto, Toronto, Ontario, Canada

${ }^{3}$ Department of Surgery, Division of General Surgery, University of Toronto, St. Michael's Hospital University of Toronto, Toronto, Ontario, Canada ${ }^{4}$ Department of Surgery, Faculty of Medicine, McGill University, Montreal, Quebec, Canada
\end{abstract}

Correspondence to Dr Joao Baptista Rezende-Neto; rezendenetoj@smh.cae000012

Received 2 May 2016 Revised 3 June 2016 Accepted 14 June 2016

\footnotetext{
To cite: Rezende-Neto JB, Leong-Poi $\mathrm{H}$, Rizoli $\mathrm{S}$, et al. Trauma Surg Acute Care Open Published Online First: [please include Day Month Year] doi:10.1136/tsaco2016-000012
}

\begin{abstract}
Background The best way to control hemorrhage from cardiac injuries is through digital occlusion followed by suture. However, this is difficult to accomplish in the emergency department (ED) setting. Generally, temporary control is obtained in advance of definitive treatment in the operating room. Despite safety and efficacy concerns, balloon Foley catheter insertion through the injury is still an option following ED thoracotomies. We developed a new device for temporary hemorrhage control in cardiac injuries and

For several decades, cardiac tamponade drainage was the only recommended procedure in the treatment of cardiac injuries. ${ }^{9} 10$ Interestingly, cardiac tamponade might have a protective effect in penetrating injuries by limiting blood loss, thus preventing exsanguination. Retrospective studies showed that the presence of tamponade was an independent predictor of survival in patients with penetrating cardiac injury. However, this finding was not confirmed on prospective investigation. ${ }^{8} 11-14$
\end{abstract} compared it to the Foley.

Methods 6 adult swine $(n=6)$ underwent full-thickness $(1.5 \mathrm{~cm})$ injury along the longitudinal axis of the right ventricle (RV). After $5 \mathrm{~s}$ of bleeding, hemorrhage control was attempted with either the device or the Foley, and blood loss quantified. Subsequently, the wound was sutured and mean arterial pressure was restored to baseline with lactated Ringer's infusion. Subsequently, another injury $2 \mathrm{~cm}$ apart in the same ventricle was managed with apparatus not employed in the first injury. The same followed in the LV totaling 4 injuries per animal, 2 in each ventricle. Intraoperative echocardiogram, laboratory test and final wound sizes assessed.

Results The device resulted in less bleeding than the Foley; RV $58.7 \pm 11.3$ vs $147.7 \pm 30.9 \mathrm{~mL}$, LV $81.7 \pm 11.9$ vs $187.5 \pm 40.3 \mathrm{~mL}(p<0.05)$. Percent change in tricuspid regurgitation was less with the device than $\mathrm{FO}, 66.6 \%$ vs $400 \%$. Mitral regurgitation increased $16 \%$ with Foley, but remained unchanged with the device. Changes in stroke volume and LV ejection fraction were less with the device than with Foley; SV $2.09 \%$ vs $12.48 \%$, left ventricular ejection fraction $0.46 \%$ vs $5.45 \%$. Foley insertion enlarged the wounds. Platelet count, complete blood count, prothrombin time, activated prothrombin time and fibrinogen decreased, whereas troponin and lactate increased compared with baseline, underscoring the magnitude of shock.

Conclusions Cardiac hemorrhage was effectively controlled with the new device. The low-profile collapsible blocking membrane interfered less with cardiac function than did the balloon of the Foley, an important asset in the context of shock.

\section{BACKGROUND}

Penetrating cardiac injuries are highly lethal. Mortality rates as high as $97 \%$ have been reported in some series. ${ }^{1-3}$ The main cause of death in patients who sustain penetrating cardiac injuries is massive bleeding. ${ }^{4}$ Previous studies showed that hemodynamic stability on arrival is associated with higher survival rates. ${ }^{6-8}$
Expeditious hemorrhage control in cardiac injury frequently entails an emergency department (ED) thoracotomy. In the setting of an ED thoracotomy, the physician will most likely encounter a hemorrhaging injury or a cardiac tamponade. ${ }^{15}$ Even though the ideal way to control hemorrhage from a cardiac injury is to digitally occlude the wound and swiftly repair it with carefully placed sutures, this is difficult to accomplish in the ED setting. Moreover, lack of experience with the definitive treatment of those injuries by ED staff poses additional problems. Therefore, temporary control of the bleeding is generally obtained in advance of the definitive treatment in the operating room (OR).

Temporary control of hemorrhage in atrial wounds is typically achieved with a vascular clamp. ${ }^{10}$ The application of this instrument generally provides enough time to transfer the patient to the OR for definitive repair. Temporary control of hemorrhage from injuries to the ventricles is significantly more challenging. The use of skin staples has been reported in several publications. ${ }^{16-19}$ This method can provide rapid hemorrhage control in the setting of multiple ventricular injuries with minimal risk of contamination from needle sticks when suturing a beating heart. However, it is frequently ineffective to control bleeding from large cardiac wounds and those caused by gunshot injurdefinitive repair of cardiac injuries should be performed with staples. Nevertheless, previous case reports showed success with the method. ${ }^{20} 21$ Additionally, there is a potential risk for enlargement of the wound and increased blood loss in the event of staple removal during the definitive suture repair of the injury. ${ }^{21}$

Despite concerns over its safety and efficacy, the use of the Foley balloon catheter to gain temporary control of the bleeding from a cardiac injury has not been abandoned, particularly following ED thoracotomies. ${ }^{5} \quad 22-26$ This technique involves the insertion of a Foley catheter through the wound followed by traction tightly positioning the balloon against the wound inside the ventricle. ies. $^{16-19}$ Moreover, it is debatable whether the 
In practice, the method and variations thereof result in suboptimal control of the bleeding and frequently lead to enlargement of the initial injury. ${ }^{27-30}$ Moreover, the balloon inevitably occupies space inside the ventricle, thereby interfering with cardiac function. ${ }^{28-30}$

We developed a new device that does not use a balloon to temporize bleeding from penetrating cardiac injuries. The device was compared with the Foley balloon catheter in an experimental model because this catheter and other instruments equipped with balloons are still used to control bleeding from cardiac injuries.

\section{METHODS}

The study was approved by the Animal Care Committee of the Li Ka Shing Knowledge Institute of St. Michael's Hospital, Toronto (protocol number ACC550).

\section{Animals}

Six adult male Yorkshire pigs $(35-37 \mathrm{~kg})$ individually housed in our animal facility, between 3 and 5 days, were used in this study. The animals were fasted the night before the procedure and were maintained at $25^{\circ} \mathrm{C}$ on 12 -hour light/dark cycles.

\section{Monitoring procedures}

Animals were anesthetized with intramuscular ketamine $(20 \mathrm{mg} /$ $\mathrm{kg})$, xylazine $(2 \mathrm{mg} / \mathrm{kg})$, and atropine sulfate $(1 \mathrm{mg} / 25 \mathrm{~kg}, 1-$ $2 \mathrm{~mL}$ ). Once anesthetized, they were mechanically ventilated $(10 \mathrm{~mL} / \mathrm{kg})$ with inhaled isoflurane $2-5 \%$ for anesthesia maintenance throughout the procedure. Pulse oximetry, ECG, and heart rate were monitored continuously. The right femoral artery was cannulated with a 14 gauge vascular catheter and mean arterial pressure (MAP) was continuously monitored (Biopac Systems, Goleta, California, USA). The right jugular vein was cannulated in similar fashion for lactated Ringer's (LR) infusion. Arterial blood samples were obtained at baseline and at the end of the experiment for arterial blood gas, complete blood count, coagulation profile, troponin, fibrinogen, and serum lactate. An intraoperative echocardiogram was performed at baseline and during temporary control of the bleeding with either the new device or the Foley.

\section{Description and operation of the new device}

The new device consists of a flexible plastic shaft, similar to a drinking straw, $\sim 3 \mathrm{~mm}$ in diameter and $12 \mathrm{~cm}$ long. The shaft passes through an opening on a $4 \mathrm{~cm}$ diameter silicone suction cup. The suction cup is adjustable to different positions along the shaft and remains stable once released. A circular low-profile collapsible blood flow blocking membrane is firmly attached to one of the extremities of the shaft (figure 1). The collapsible membrane is made of natural rubber; it is $3.5 \mathrm{~cm}$ in diameter and $1 \mathrm{~mm}$ in width. This membrane is not an inflatable balloon; it expands on the basis of the elastic properties of the rubber and the pressure created by the blood inside the ventricle.

The new device is deployed by introducing the collapsible blood flow blocking membrane through the cardiac injury, and subsequently sliding the silicone suction cup down toward the injury on the surface of the heart (figure 2A). Thereby, abutting the membrane to the undersurface of the ventricle wall creating a firm seal (figure 2B). The device is seamlessly removed from the ventricle by sliding the suction cup upward along the plastic shaft, away from the surface of the heart, and pulling the device out of the wound. In contrast, the Foley balloon requires complete deflation before removal. The collapsible property of the inner blocking membrane maintains continuous control of the

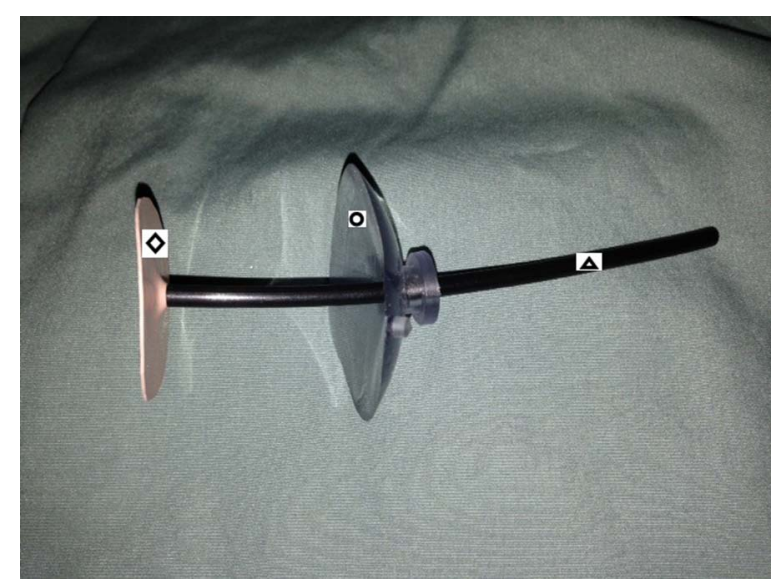

Figure 1 Components of the device: $\mathbf{O}$ silicone suction cup; flexible shaft; $\forall$ low-profile collapsible blood flow blocking membrane.
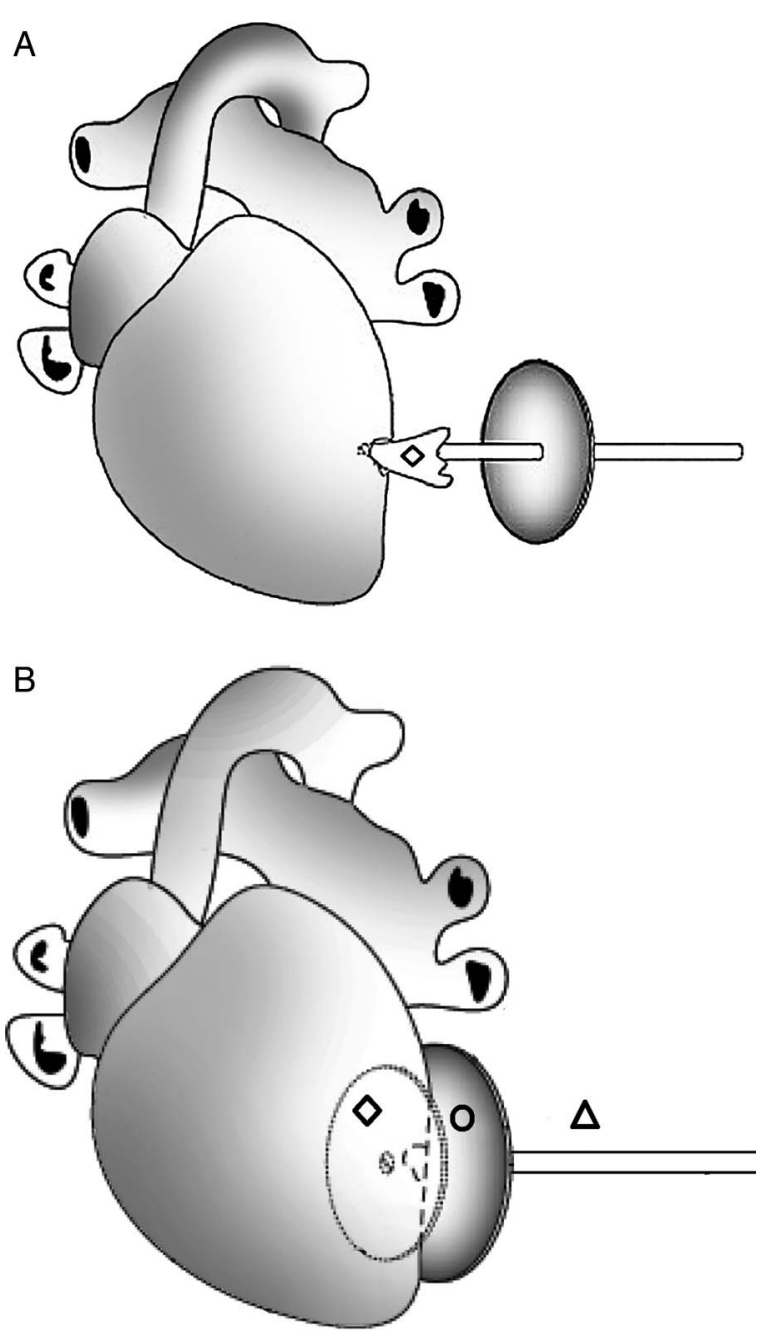

Figure 2 (A) Insertion of the device through the ventricular injury. $\checkmark$ Low-profile collapsible blood flow blocking membrane. (B) This figure depicts the device fully deployed. $\$$ Low-profile collapsible blood flow blocking membrane deployed inside the ventricle sealing the injury. The silicone suction cup positioned against the outer surface of the heart overlying the injury and abutting the blood flow blocking membrane. $\mathbf{\Delta}$ The flexible shaft. 
bleeding as the device is being removed, facilitating suture repair of the wound and preventing stitching through the membrane.

\section{Cardiac injury and hemorrhage control}

A median sternotomy was performed and the pericardial sac opened to expose the heart. Subsequently, a baseline intraoperative echocardiogram (Phillips HD 15 Ultrasound System, Andover, Massachusetts, USA) was performed by an experienced cardiologist prior to the injury. A suction catheter and preweighed dry sponges were placed in the mediastinum to collect the bleeding. Two scalpel blades number 22 (Integra Miltex Medical Instruments, York, Pennsylvania, USA) were securely taped in parallel (Elastoplast EAB, Brisbane, Australia) to a scalpel handle, producing a cutting surface measuring exactly $1.5 \mathrm{~cm}$ in length and $1.5 \mathrm{~cm}$ in depth. The remaining cutting surfaces of the blades were completely covered with tape to prevent extension of the injury. The standardized full-thickness $(1.5 \mathrm{~cm})$ injury was initially made in the right ventricle (RV). To ensure that a transmural injury was performed and not just a puncture wound, the exposed portions of the blades were completely introduced inside the ventricle up to the edge of the tape. The injury was allowed to bleed freely for $5 \mathrm{~s}$. Subsequently, hemorrhage control was obtained with either the new device or the Foley catheter, never both at the same time. The balloon of the Foley catheter (18 Fr) (C.R. Bard, Murray Hill, New Jersey, USA) was filled with $10 \mathrm{~mL}$ of distilled water as per the manufacturer's instructions and pulled against the wound inside the ventricle. Removal of the Foley catheter was achieved by deflating the balloon and pulling the catheter out of the ventricle.

Blood loss was assessed after complete control of the bleeding by measuring the volume of blood aspirated from the mediastinum, and the difference in weight between blood-soaked sponges minus the weight of the pre-weighed dry sponges.

Once the blood loss volume was quantified, another intraoperative echocardiogram was performed with either the new device or the Foley still inside the ventricle. Subsequently, the apparatus used in temporary hemorrhage control was appropriately removed and the injury repaired with 2-0 silk sutures (SOFSILK, Covidien, Sunnyvale, California, USA). The ensuing hypotension was treated with the infusion of LR to maintain MAP stable at baseline levels $\pm 5 \mathrm{~mm} \mathrm{Hg}$ for $5 \mathrm{~min}$. Thereafter, another injury was created $2 \mathrm{~cm}$ apart from the previous one along the longitudinal axis of the ventricle. Temporary control of the bleeding was achieved with the apparatus not employed in the first injury, thus ensuring equivalent management of the injuries. The same procedures were performed in the left ventricle (LV) totaling four injuries per animal, two in each ventricle. In subsequent animals, the apparatus used to control the bleeding from the first injury alternated between the new device and the Foley catheter. Therefore, both methods were used under the same conditions, the same number of times. In the aforementioned context, the Foley catheter was randomly selected for temporary hemorrhage control of the first injury in the first animal.

The animals were euthanized by the vivarium staff with the injection of T-61 euthanasia solution at the end of the experiment (Merck Animal Health Intervet Canada Corp., Kirkland, Quebec, Canada). Subsequently, the size of each injury was measured with a ruler after careful removal of the sutures.

\section{Statistical analysis}

Hemodynamic and laboratory data are reported as mean \pm SD. Echocardiographic data are reported as percent change from baseline values. The Student's t-test was used to analyze data comparison between the two methods; $\mathrm{p}<0.05$ was considered statistically significant (GraphPad Prism V.6.00 for Windows, GraphPad Software, La Jolla, California, USA).

\section{RESULTS}

The mean weight of the animals was $36.1 \pm 0.3 \mathrm{~kg}$. Baseline MAP was $82.2 \pm 7.8 \mathrm{~mm} \mathrm{Hg}$ cardiac injuries resulted in a significant decrease in MAP $68.5 \pm 12.1 \mathrm{~mm} \mathrm{Hg}(\mathrm{p}=0.001)$.

Temporary control of the bleeding with the new device resulted in significantly less bleeding than with the Foley catheter, in both right ventricular (RV) and left ventricular (LV) injuries; respectively RV $58.7 \pm 11.3$ vs $147.7 \pm 30.9 \mathrm{~mL}$ $(\mathrm{p}=0.022)$, and LV $81.7 \pm 11.9$ vs $187.5 \pm 40.3 \mathrm{~mL}(\mathrm{p}=0.03)$.

The intraoperative echocardiogram performed during the use of the new device showed less change in echocardiographic parameters than with the Foley catheter, compared with baseline values (table 1 ).

The final size of the injuries was significantly larger when the Foley catheter was used to control bleeding compared with the new device; respectively $1.53 \pm 0.02$ vs $1.8 \pm 0.1 \mathrm{~cm}(\mathrm{p}=0.002)$.

Results of the laboratory tests performed during the study comparing baseline samples to those obtained at the end of the experiment are shown in table 2 .

There were no statistically significant differences in the arterial blood gas parameters.

\section{DISCUSSION}

Digital compression is the best way to control hemorrhage from cardiac injuries. However, it is mostly effective if used concomitantly with suture repair of the wound. In this study, we tested a new device designed to provide temporary control of bleeding from ventricular injuries. The results showed that the new device adequately controlled the bleeding and was more effective than the Foley catheter in several aspects. Up front, the new device resulted in significantly less bleeding than the insertion of the Foley catheter. The silicone suction cup compressed the wall of the ventricle against the blood flow blocking membrane and functioned as an external abutment to the pressure inside the ventricle (figure 3). The higher the pressure inside the ventricle, the stronger was the force pushing against the membrane and the suction cup. This helped to improve the occlusion of the wound. The concave and pliable silicone suction cup adapted to a large area on the surface of the ventricle, inducing better distribution of the pressure across the ventricular wall.

The operation of the new device does not require balloon insufflation to control bleeding. Those features may have a favorable impact in the setting of cardiac injuries. Insufflation of the Foley balloon unavoidably occupies space inside the ventricle that would otherwise be filled with blood, thus provoking hemodynamic derangement. ${ }^{28-30}$ Furthermore, insufflation of a

Table 1 Percent change of intraoperative echocardiographic parameters from baseline values

\begin{tabular}{lcc}
\hline Parameters & New device (\%) & Foley catheter (\%) \\
\hline TR (increase) & 66.6 & 400 \\
MR (increase) & 0 & 16 \\
Stroke volume (decrease) & 2.09 & 12.48 \\
LVEF (decrease) & 0.46 & 5.45 \\
\hline LVEF, left ventricular ejection fraction; MR, mitral regurgitation; TR, tricuspid \\
regurgitation.
\end{tabular}


Table 2 Laboratory parameters at baseline and after the cardiac injuries

\begin{tabular}{lccl}
\hline Parameters & Baseline values & Final values & $\mathbf{p}$ Value* \\
\hline $\mathrm{RBC} \times 10^{12} / \mathrm{L}$ & $5.3 \pm 0.3$ & $3.9 \pm 0.3$ & 0.0003 \\
Hemoglobin, g/dL & $91 \pm 4.8$ & $66.5 \pm 4.5$ & 0.0002 \\
Hematocrit, $\mathrm{L} / \mathrm{L}$ & $0.3 \pm 0.01$ & $0.2 \pm 0.02$ & 0.0001 \\
Platelets $\times 10^{9} / \mathrm{L}$ & $271.5 \pm 30.9$ & $223.2 \pm 16.1$ & 0.037 \\
Fibrinogen, g/dL & $1.6 \pm 0.2$ & $1.1 \pm 0.1$ & 0.01 \\
$\mathrm{PT}$, seconds & $14.6 \pm 0.4$ & $15.9 \pm 0.3$ & 0.001 \\
APTT, seconds & $10.6 \pm 0.4$ & $12.18 \pm 0.2$ & 0.005 \\
Lactate, mmol/L & $2.6 \pm 0.3$ & $4.8 \pm 0.9$ & 0.03 \\
Troponin, ng/mL & $0.2 \pm 0.005$ & $0.9 \pm 0.07$ & 0.001 \\
\hline * $\mathrm{p}<0.05$ was considered statistically significant. & \\
APTT, activated prothrombin time; PT, prothrombin time; RBC, red blood cell.
\end{tabular}

balloon inside the ventricle can also interfere with the normal function of the valve apparatus, given that the pressure gradient across this structure is what causes the opening and closing of the valve. ${ }^{31-33}$ A previous study in an isolated swine heart model using a high-speed digital video camera showed that altered preload significantly impacts the normal function of the mitral subvalvular apparatus. ${ }^{32}$ Therefore, the significant increase in tricuspid and mitral valve regurgitation with the Foley catheter, compared with the new device, could be related to a combination of a change in the preload and abnormal function of the subvalvular apparatus caused by the balloon. Moreover, previous work has shown that dysfunction of the

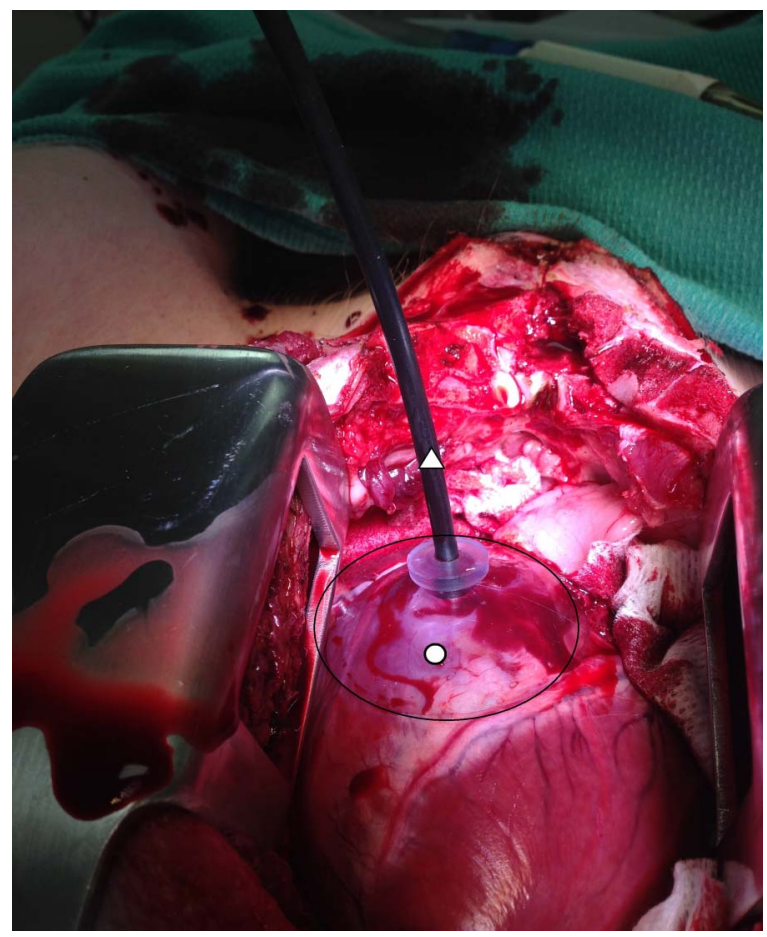

Figure 3 Intraoperative photograph depicts the new device temporizing hemorrhage in a right ventricular injury in the beating heart. Hemorrhage is controlled without traction to the flexible shaft.

- The silicone suction cup is positioned against the ventricle over the site of the injury. The low-profile collapsible blood flow blocking membrane sealing the injury from inside the ventricle (not shown). A The flexible shaft. mitral subvalvular apparatus interferes with the normal threedimensional geometry of the ventricle resulting in LV systolic dysfunction. $^{34}$ In keeping with that information, our results showed that the percent change of reduction in SV with the Foley catheter was almost six times higher than with the new device. Similarly, the percent change of reduction in left ventricular ejection fraction was almost 12 times higher with the Foley than with the new device. Essentially, the low-profile collapsible blocking membrane interfered less with cardiac function than did the balloon of the Foley catheter in the beating heart. This is all important in the setting of penetrating cardiac injuries. $^{28} 29$

Cardiac wound size assessment at the end of the experiment showed that the use of the Foley catheter augmented the injuries. We hypothesize that the upward traction applied to the Foley catheter to position the balloon against the inner surface of the ventricle and effectively temporize the bleeding was the predominant cause for the increase in the wound size. In contrast, the new device operates with the application of downward pressure toward the surface of the heart. Pragmatically, it is conceivable that excessive traction of the Foley catheter can easily occur in the frenetic environment of a trauma center during attempts to gain control of the hemorrhage in a beating heart. Actually, this complication has been previously reported in the clinical setting. ${ }^{28} 29$

This study has limitations most inherent to experimental models. We attempted to use the fewest number of animals necessary to obtain meaningful data; hence, similar conditions were created for comparison of the device to the Foley catheter in each animal. Even though we standardized the injuries and used each method in similar situations and the same number of times, the physiological derangement of the animals following each injury was inevitable. Similarly, it is difficult to translate the hemodynamic consequences of inflating a $10 \mathrm{~mL}$ balloon inside the ventricle of a $35 \mathrm{~kg}$ animal to a $70 \mathrm{~kg}$ human. Furthermore, it is difficult to ascertain the clinical benefit of the slight, albeit statistically significant, decrease in the final wound size between the two methods. A more detailed assessment of the wound size, a larger injury or a different injury mechanism, for example, penetrating gunshot wounds, could potentially reveal more meaningful results. Nevertheless, the magnitude of the injuries sustained by the animals was represented by the significant derangements in the coagulation profile, lactate, and troponin levels at the end of the experiment. Considering the acute nature of the experiment and the severity of the bleeding experienced by the animals, we felt that histopathological assessment to confirm the extent of the injuries was unnecessary. Finally, to further understand the usefulness of the new device, it is important that physicians who are less experienced with the treatment of penetrating cardiac injuries also test it in experimental models.

In summary, bleeding from ventricular injury was effectively controlled with the new device. The low-profile collapsible blocking membrane interfered less with cardiac function than did the balloon of the Foley catheter. Considering that the deployment of the new device did not involve upward traction, the risk of increasing the size of the wound was minimized. Our ongoing research involves exploring new materials in the production of the device.

Contributors JBR-N invented the new device; and performed the surgical procedures. HL-P performed intraoperative echocardiogram. JBR-N, SR and AB participated in the development of the animal model. All the authors contributed to the data analysis; and in the writing, editing and critical revision of the manuscript. 
Funding JBR-N provided personal financial support for the development of the new device. Animal experiments were funded by the Academic Enrichment Fund from the Division of General Surgery.

Competing interests JBR-N has filed a provisional patent application for the device described in this study.

Provenance and peer review Not commissioned; externally peer reviewed.

Open Access This is an Open Access article distributed in accordance with the Creative Commons Attribution Non Commercial (CC BY-NC 4.0) license, which permits others to distribute, remix, adapt, build upon this work non-commercially, and license their derivative works on different terms, provided the original work is properly cited and the use is non-commercial. See: http://creativecommons.org/ licenses/by-nc/4.0/

\section{REFERENCES}

1 Campbell NC, Thomson SR, Muckart DJ, et al. Review of 1198 cases of penetrating cardiac trauma. Br J Surg 1997;84:1737-40.

2 Demetriades $D$, van der Veen BW. Penetrating injuries to the heart: experience over two years in South Africa. J Trauma 1983;23:1034-41.

3 Rodrigues AJ, Furlanetti LL, Faidiga GB, et al. Penetrating cardiac injuries: a 13-year retrospective evaluation from a Brazilian trauma center. Interact Cardiovasc Thorac Surg 2005:4:212-15.

4 Asensio JA, Murray J, Demetriades $D$, et al. Penetrating cardiac injuries: a prospective study of variables predicting outcomes. I Am Coll Surg 1998;186:24-34.

5 Wilson SM, Au FC. In extremis use of a Foley catheter in a cardiac stab wound. J Trauma 1986:26:400-2.

6 Asensio JA, Soto SN, Forno W, et al. Penetrating cardiac injuries: a complex challenge. Injury 2001;32:533-43.

7 Rhee PM, Foy H, Kautmann C, et al. Penetrating cardiac injuries: a population-based study. J Trauma 1998;45:366-70.

8 Tyburski JG, Astra L, Wilson RF, et al. Factors affecting prognosis with penetrating wounds to the heart. J Trauma 2000;48:587-90. doi:discussion 590-1

9 Werner OJ, Sohns C, Popov AF, et al. Ludwig Rehn (1849-1930): the German surgeon who performed the worldwide first successful cardiac operation. J Med Biogr 2012;20:32-4.

10 Kang N, Hsee L, Rizoli S, et al. Penetrating cardiac injury: overcoming the limits set by nature. Injury 2009;40:919-27.

11 Moreno C, Moore EE, Majure JA, et al. Pericardial tamponade: a critical determinant for survival following penetrating cardiac wounds. J Trauma 1986;26:821-5.

12 Asensio JA, Berne JD, Demetriades $D$, et al. One hundred and five penetrating cardiac injuries. A 2-year prospective evaluation. J Trauma 1998;44:1073-82.

13 Buckman RF, Badellino MM, Mauro LH, et al. Penetrating cardiac wounds: prospective study of factors influencing initial resuscitation. J Trauma 1993;34:717-25.

14 Degiannis E, Loogna P, Doll D, et al. Penetrating cardiac injuries: recent experience in South Africa. World I Surg 2006;30:1258-64.
15 Cothren CC, Moore EE. Emergency department thoracotomy for the critically injured patient: objectives, indications and outcomes. World I Emerg Surg 2006;1:4.

16 Bowman MR, King RM. Comparison of staples and sutures for cardiorrhaphy in traumatic puncture wounds of the heart. J Emerg Med 1996;14:615-18.

17 Chapman GT, Frame SB. Repair of penetrating cardiac injury using skin staples. Tenn Med 1996:89:249-51.

18 Macho JR, Markison RE, Schecter WP. Cardiac stapling in the management of penetrating injuries of the heart: rapid control of hemorrhage and decreased risk of personal contamination. J Trauma 1993;34:711-15.

19 Mayrose J, Jehle DV, Moscati $R$, et al. Comparison of staples versus sutures in the repair of penetrating cardiac wounds. J Trauma 1999;46:441-3. discussion 443-4.

20 Asensio JA, Stewart BM, Murray J, et al. Penetrating cardiac injuries. Surg Clin North Am 1996;76:685-724.

21 Monneuse 0 , Al-Ahmed K, Ahmed N. Definitive repair of a stab wound to the right ventricle with skin staples in emergency. Wounds 2008;20:219-20.

22 Wall MJ Jr, Tsai P, Mattox KL. Heart and thoracic vascular injuries. In: Mattox KL, Moore EE, Feliciano DV, eds. Trauma. New York, NY: McGraw Hill Medical, 2013:485-511.

23 Feliciano DV. Cardiac, great vessel, and pulmonary injuries. In: Rasmussen TE, Tai NRM, eds. Rich's vascular trauma. Philadelphia, PA: Elsevier, 2016:73-99.

24 Eastridge BJ, Blackbourne LH, Rasmussen $T$, et al. Damage control surgery. In: Savitsky E, Eastridge B, eds. Combat casualty care: lessons learned from OEF and OIF. Frederick, MD: Office of the Surgeon General, 2012:165-224.

25 Jacobs LM, Luk SS. The cardiovascular system. In: Jacobs LM, Luk SS, eds. American College of Surgeons Committee on Trauma Advanced Trauma Operative Management: surgical strategies for penetrating trauma. Woodbury, CT: Ciné-Med, Inc, 2010:239-54

26 Tesoriero R. Cardiac injury. In: Scalea TM, ed. The shock trauma manual of operative techniques. New York, NY: Springer-Verlag, 2015:173-94.

27 Attar S, Suter CM, Hankins JR, et al. Penetrating cardiac injuries. Ann Thorac Surg 1991;51:711-15; discussion 715-16.

28 Pham SM, Johnson R, Durham SJ, et al. Hemodynamic consequences of Foley catheter control in experiment penetrating cardiac wounds. Surg Gynecol Obstet 1989;169:247-50.

29 Sen A, Anderson L. Foley catheter haemostasis for penetrating cardiac wounds: the need for caution. Arch Emerg Med 1993;10:385-6.

30 Tokur M, Ergin M, Kurkçuoglu IC, et al. A pilot experimental study of a catheter to facilitate treatment for penetrating cardiac injury. Turk J Med Sci 2014;44:935-40.

31 Araki Y, Usui A, Kawaguchi O, et al. Pressure-volume relationship in isolated working heart with crystalloid perfusate in swine and imaging of the valve motion. Eur I Cardiothorac Surg 2005;28:435-42.

32 Saito S, Araki Y, Usui A, et al. Mitral valve motion assessed by high-speed video camera in isolated swine heart. Eur I Cardiothorac Surg 2006;30:584-91.

33 Stoddard MF, Pearson AC, Kern MJ, et al. Influence of alteration in preload on the pattern of left ventricular diastolic filling as assessed by Doppler echocardiography in humans. Circulation 1989;79:1226-36.

34 Yun KL, Niczporuk MA, Sarris GE, et al. Importance of mitral subvalvular apparatus in terms of cardiac energetics and systolic mechanics in the ejecting canine heart. J Clin Invest 1991;87:247-54. 